\title{
Visual fields in a chloroquine treatment
}

This article was published in the following Dove Press journal:

Clinical Optometry

30 September 2014

Number of times this article has been viewed

\author{
María del Carmen García- \\ Domene ${ }^{1,2}$ \\ María Amparo Díez-Ajenjo ${ }^{1,3}$ \\ María Dolores de $\mathrm{Fez}^{4}$ \\ María José Luque ${ }^{2}$ \\ 'Fisabio Oftalmología Médica, \\ València, '² Departament d'Òptica, \\ Facultat de Física, Universitat \\ de València, Burjassot, ${ }^{3}$ Clínica \\ Optométrica Fundación Lluís Alcanyís, \\ Valencia, ${ }^{4}$ Departamento de Óptica, \\ Farmacología y Anatomía, Universidad \\ de Alicante, Alicante, Spain
}

Correspondence: María del Carmen García-Domene Fisabio Oftalmología Médica, Intersección Pió Baroja-General Avilés, s/n 46015, Valencia, Spain

Tel +34962328100

Fax +34962328102

Email m.carmen.garcia-domene@uv.es
Purpose: In this study, we describe contrast sensitivity losses in the visual field of a patient affected by chloroquine toxicity, measured with stimuli favoring different visual mechanisms. We have compared these results with those of other, usual clinical tests.

Methods: The vision of a patient who underwent ten years of chloroquine treatment was analyzed by a battery of clinical tests: visual acuity (VA), Amsler's grid, Farnsworth-Munsell 100hue test, spectral domain optical coherence tomography, multifocal electroretinogram (ERG), white-on-white and red-on-white Humphrey perimetries, chromatic and achromatic contrast sensitivity perimetry tests, and fluorescein angiography. Measurements were taken just before the cessation of the treatment, and 6 months later.

Results: The subjective visual perception of the patient was worse (in comparison with the rest of the visual field at the time of the first visit) in the center of the visual field, and was impaired on the second visit. Although standard automated perimetry (SAP) was practically normal and ERG results did not worsen with time, VA, Amsler's grid, and visual fields with stimuli favoring the magnocellular and chromatic pathways signalled progressive loss of visual function.

Conclusions: Standard tests such as SAP or ERG may not detect visual field losses or progression of existing visual losses in a case of chloroquine toxicity, whereas tests evaluating contrast sensitivity with stimuli favoring different visual mechanisms may be more sensitive.

Keywords: visual field, chloroquine, retinal toxicity, retinal damage, color perception

\section{Introduction}

The antimalarial drugs chloroquine and hydroxychloroquine have a well-established beneficial role in the treatment of rheumatoid arthritis, systemic lupus erythematosus, ${ }^{1,2}$ and other connective tissue and skin disorders, but their use has been associated with the development of retinal toxicity. ${ }^{3}$ This ocular toxicity was first described in the literature as early as 1957 , by Cambioggi et al. ${ }^{4}$

In the early stages of chloroquine retinal toxicity, there appear psychophysical signs of retinopathy, characterized by a paracentral scotoma on threshold visual field testing, without any observable fundus change; also, possibly, by the presence of color vision defects. ${ }^{5}$ Advanced retinopathy may show the typical "bull's eye" maculopathy associated with impaired visual acuity and central visual field defects. ${ }^{6}$ These retinal changes are irreversible and may progress even after cessation of medication. ${ }^{7,8}$ When the recommended dose of $3 \mathrm{mg}$ chloroquine and $6.5 \mathrm{mg}$ hydroxychloroquine per kilogram ideal body weight is not exceeded, the risk of retinopathy is low, but it increases with prolonged treatment. ${ }^{9}$ 
The American Academy of Ophthalmology (AAO) recommends screening intervals after a baseline examination for both antimalarials, because of the anticipated risk to the patient. ${ }^{10}$ The proposed screening routine includes ophthalmological examination, far and near best-corrected visual acuities, and visual field testing (Humphrey 10-2 perimetry). Optional tests include spectral domain optical coherence tomography (SDOCT), fundus autofluorescence (FAF), and multifocal electroretinogram (mfERG). However, there is only a limited number of studies that compare the sensitivity and specificity of these procedures relative to automated visual field testing. The optimal test or combination of tests is still unknown, ${ }^{11,12}$ and the relative efficiency of quantitative tests like Humphrey perimetry, ${ }^{10-12}$ mfERG or SDOCT is still under debate. $^{13}$

Other alternative tests for detecting this form of retinopathy have been proposed. Xiaoyun et a $1^{14}$ recommended analysis of the retinal nerve fiber layer thickness with the GDx VCC scanning laser polarimeter, in order to detect early retinal damage. Recent studies show that chloroquine and hydroxychloroquine induce impairment in the sensitivity of the central retina that can be detected by microperimetry before retinal damage is visible. ${ }^{15,16}$

Although the guidelines of the AAO pay particular attention to structural damage and to the achromatic mechanism, defects in color vision are known to occur with antimalarial treatment that causes retinal toxic damage, ${ }^{5,17,18}$ which may precede fundus alteration. ${ }^{18}$ However, reported changes may be subtle, and frequently are not detected by conventional color vision testing. ${ }^{19-24}$ The earliest color anomalies resulting from retinal toxicity occur along the blue-yellow (ie, tritan) direction of color space, while redgreen (protan) defects occur in more advanced cases. . $^{5,26}$ For this reason, color vision tests exploring both opponent chromatic mechanisms, and capable of detecting slight discrimination changes, are needed to adequately characterize these patients. Vu et $\mathrm{al}^{27}$ compared different clinical color vision tests on patients with established retinopathy and normal patients with rheumatic diseases and found that Standard Pseudoisochromatic Plates Part 2 had the best test characteristics for screening, yielding a sensitivity of $93 \%$ and a specificity of $88 \%$. Neubauer et al ${ }^{5}$ showed that for advanced bull's eye, color vision testing has $75 \%$ sensitivity and $91 \%$ specificity, even when oculograms are normal. They conclude that psychophysical tests are more effective for detecting alterations caused by chloroquine retinotoxicity.

However, most clinical color vision tests assess only central vision. It could be useful to compare the state of the different visual pathways of subjects under chloroquine treatment at different locations of the visual field. The first stages of visual perception are mediated by three parallel pathways of magnocellular, parvocellular, and koniocellular origin, respectively, each encoding different visual information. Achromatic information is processed by both the magnocellular and parvocellular pathways, although the first mechanism is more sensitive to low spatial frequency and high temporal frequency stimuli, and mediates movement perception. The second mechanism is most sensitive to stationary stimuli, and mediates shape perception and vision of spatial detail. ${ }^{28-32}$ Chromatic information is processed by the red-green and blue-yellow opponent mechanisms, respectively, of parvocellular and koniocellular origin. ${ }^{33}$ Though in clinical practice more attention is paid to losses in the achromatic mechanisms, acquired anomalies in the chromatic mechanisms may seriously impair perception (for some demonstrative examples of the perception of subjects with damage in the chromatic mechanisms, see Capilla et a $\left.{ }^{34}\right)$.

In this study we present the case of an adult woman in a ten-year chloroquine treatment, just before cessation of the medication, and 6 months later. To take into account the effect of damage in different visual mechanisms, we have analyzed contrast sensitivity throughout the visual field, using stimuli with different chromatic and spatiotemporal characteristics. Measurements have been carried out using a computerized perimeter: the anthropometric test devices (ATD) Multichannel Functional Test, developed by the University of Valencia and INDO Internacional SA (Barcelona, Spain) (US Patent: 7.641.344 B2; Spanish Patent: 2246174). ${ }^{35,36}$

\section{Case report}

\section{Patient}

The patient, a 59-year-old female, was diagnosed 10 years ago with ankylosing spondylitis and treated since then with chloroquine $(150 \mathrm{mg} /$ day). She was otherwise healthy, with no antecedents of systemic or ophthalmological diseases. One year ago, she began to experience difficulty in reading. She also reported problems in far vision, especially in contrast discrimination. This patient was comparatively young when she began treatment and has not taken other drugs during this period. 


\section{Methodology}

The procedures adhered to the tenets of the Declaration of Helsinki; the patient read and signed an informed consent form before any measurement.

The patient underwent the following test battery: tonometry, optical coherence tomography, angiography, measurement of best-corrected visual acuity (BCVA) with the Early Treatment Diabetic Retinopathy Study (ETDRS) test, color vision testing with the Farnsworth-Munsell 100-hue test under D-65 Illuminant, Amsler's grid, mfERG, Humphrey 24-2 visual field (with and without red filter), and ATD visual fields. All the measurements were taken on the first visit and 6 months after the chloroquine treatment was interrupted.

Farnsworth-Munsell 100-hue test results are shown first as polar plots drawn with Farnsworth's criterion and then smoothed with the procedure proposed by Dain and Birch, ${ }^{37,38}$ to show the regions of relatively greater sensitivity losses. Total error scores (TES) were computed by summing the partial error scores for every cap. Total partial error scores were computed for the red-green (RG) axis using caps 13-33 and 55-75, and for the blue-yellow (BY) axis using caps 1-12, 34-54, and $76-85$, as recommended by Smith et al. ${ }^{39}$ Normality limits for TES were those derived by Kinnear and Sahraie. ${ }^{40}$
The ATD Multichannel Functional Test has been described in detail in Anton and co-workers. ${ }^{35}$ Stimuli were generated on a colorimetrically-characterized and gamma-corrected 17-inch LG Flatron F700P CRT monitor, driven by a 12-bit video controller (Bits\#; Cambridge Research Systems, Rochester, UK). Measurements were carried out in a darkened room. Patients were initially shown a spatially-uniform achromatic field, covering a $60^{\circ}$ horizontal by $40^{\circ}$ vertical fovea-centered area, and were asked to fixate upon a central 0.5 degree wide black cross during the measurement session. After an adaptation period of 30 seconds, contrast sensitivity was assessed in 21 locations using flickering achromatic RG and BY gratings with Gaussian smoothed borders (see Figure 1 for a description of the spatial and temporal profiles of the stimuli, an image of the four stimuli used in the study, and the spatial distribution of testing points). The screen was placed at $25 \mathrm{~cm}$ from the patient, who wore a $4.00 \mathrm{D}$ addition, to avoid accommodation. The subject was instructed to press a button if any variation from the background was detected at any point of the visual field. Thresholds were determined by an interleaved stepwise threshold algorithm. At each trial, the testing point was changed at random. In
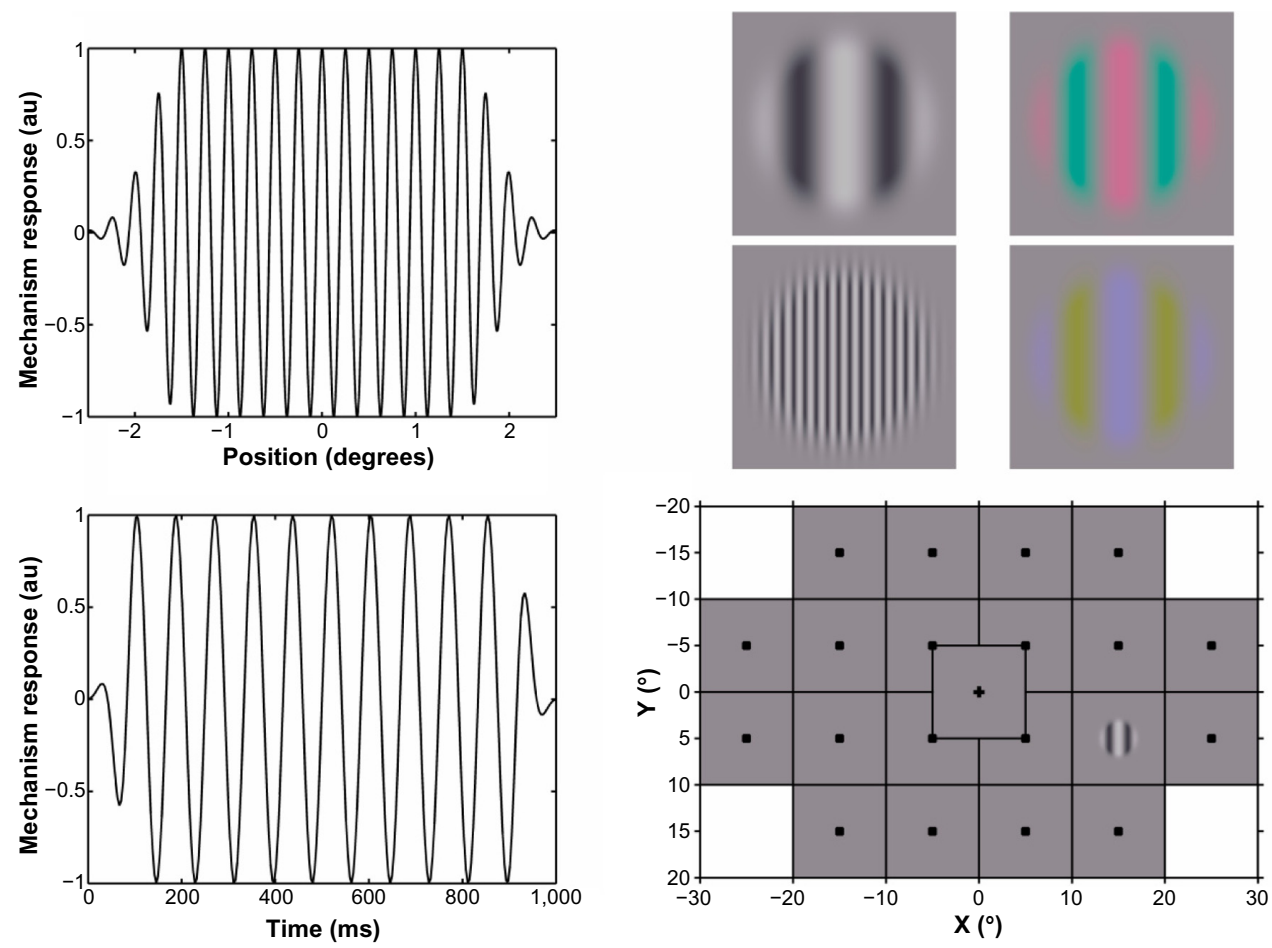

Figure I Typical spatial and temporal profiles of the stimuli generated in the ATD perimeter.

Notes: Left: example of spatial (top) and temporal (bottom) profile of the kind of stimulus used in the ATD perimeter. Right (top): single frames of the achromatic, red-green, and blue-yellow stimuli used in the measurements. The low spatial frequency achromatic stimulus corresponds to $A(M)$ and the high-frequency one to $A(P)$. Right (bottom): distribution of testing points in the visual field. At location $\left(15^{\circ},-5^{\circ}\right)$, a sample stimulus is shown, to give an indication of relative size. 
Table I Farnsworth-Munsell Score

\begin{tabular}{lll}
\hline & First visit & Second visit \\
\hline OD & & \\
TES & $184(P<0.05)$ & $172(P<0.05)$ \\
RG & 92 & 98 \\
BY & 92 & 74 \\
OS & & \\
TES & $200(P<0.05)$ & $180(P<0.05)$ \\
RG & 112 & 68 \\
BY & 88 & 112 \\
\hline
\end{tabular}

Notes: TES: total error score; RG: score for the red-green mechanism; BY: score for the blue-yellow mechanism. The RG and BY scores were computed following Smith et al. ${ }^{39}$ The normality limits used were those derived by Kinnear and Sahraie. ${ }^{40}$ Abbreviations: OD, right eye; OS, left eye.

the first trial, at a given testing point, the stimulus had the maximum amplitude achievable by the CRT. If this stimulus was detected, amplitude was divided by 2 at that point in the next trial, and continued decreasing in this way until the subject failed to detect the stimulus. The staircase was then reversed and amplitude increased by $2^{1 / 2}$ for the next presentation, and continued increasing in this way until the test was again detected. This triggered a second reversal, and amplitude was divided by $2^{1 / 4}$, and so on. The criteria for exiting the staircase procedure at a given point were either totaling four reversals or 20 presentations, whichever came first. Among the stimuli presentations, up to 16 false positive trials and 10 false negative trials were also randomly interleaved. Additionally, each session included up to 8 fixation losses catch trials, presented in the blind spot location previously estimated for the subject.

OD

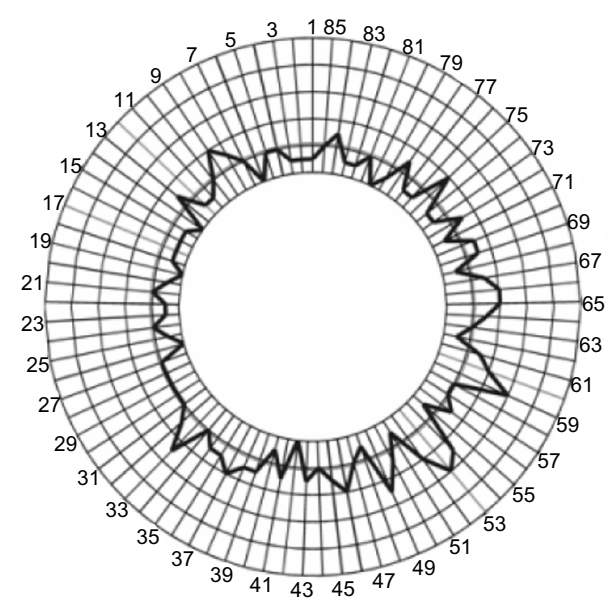

To favor different visual mechanisms, particular chromaticities and spatiotemporal frequencies were chosen (see Anton et $\mathrm{a}^{35}$ for a detailed discussion). To evaluate the achromatic mechanism A, we used a stimulus favoring the magnocellular pathway (A: 0.5 cycles per degree [cpd], $12 \mathrm{~Hz}$ ) and other stimulus stimulating the parvocellular pathway (A: $4 \mathrm{cpd}$, $2 \mathrm{~Hz}$ ). The RG and BY chromatic mechanisms, putatively mediated by the parvocellular and koniocellular pathways, respectively, were evaluated by stimuli modulated along the RG and BY directions of the Derrington Krauskopf Lennie (DKL) color space, ${ }^{41,42}$ with a spatial frequency of $0.5 \mathrm{cpd}$ and a temporal frequency of $2 \mathrm{~Hz}$ (RG: $0.5 \mathrm{cpd}, 2 \mathrm{~Hz}$; BY: $0.5 \mathrm{cpd}$, $2 \mathrm{~Hz}$ ). Results were compared with a normal database of the same age range as the patient, and a total deviation map and a pattern deviation map, following the usual definitions, were plotted, with $P$-values codified in grayscale.

\section{Results}

\section{First visit}

In the first visit intraocular pressure (IOP) was $13 \mathrm{mmHg}$ in both eyes. Both the angiography and the optical coherence tomography (OCT) were normal, but in the Amsler's grid, the patient referred a diffuse central zone. BCVA values in LogMAR scale were 0.1 and 0.14 , for the right and the left eyes, respectively. The Farnsworth-Munsell 100-hue test results showed significant generalized losses in all the color space (see Table 1 and Figure 2), with TES outside normal limits $(P<0.05)$. The smoothed plots computed using Dain and Birch's criterion ${ }^{37,38}$ (Figure 3) showed that, in both eyes, the defect appeared to be nonselective, affecting both

OS

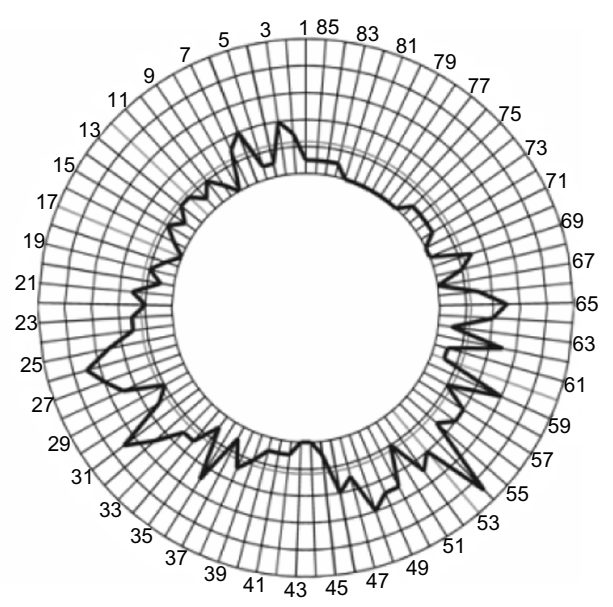

Figure 2 Results for Farnsworth-Munsell 100-hue test on the first visit. Abbreviations: OD, right eye; OS, left eye. 
the RG and the BY mechanisms, although, in the left eye, a slightly greater loss in the RG than in the BY mechanism was suggested.

The visual field with the Humphrey white-on-white (SAP) was normal (Figure 4). There is not a normative database for the red-filter Humphrey perimetry, but the results show a local sensitivity decrease at the center of the visual field (Figure 5). The mfERG was consistent with the result of the red-on-white perimetry, since it was also altered in the center (results of wave P1 analysis, see Table 1).

The results of the perimetry tests for the four visual mechanisms on the first visit are shown in Figures 6 and 7. In all cases, the patient presented absolute sensitivity losses, more marked in the center of the visual field. The pattern deviation map showed relative losses in the central field, for both achromatic mechanisms, and isolated losses in the periphery for both chromatic mechanisms. Globally, the achromatic parvocellular pathway appeared to be the less affected.

\section{Second visit}

At the second visit, BCVA was 0.14 and 0.2. IOP was $12 \mathrm{mmHg}$ and $13 \mathrm{mmHg}$, for the right and left eye, respectively. In the OCT, we detected foveolar atrophy, and, in the Amsler's grid, the patient reported a larger blurred area in the central region than on the first visit. The FarnsworthMunsell 100-hue test (Figure 8) showed that TES remained outside normal values. With the right eye, the defect seemed to selectively affect the RG mechanism; in the left eye, the

\section{OD}

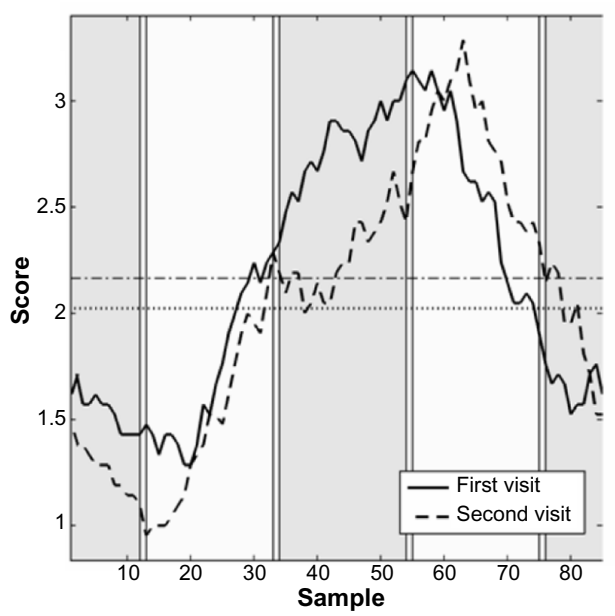

BY mechanism was the more affected (see the Dain-Birch diagram and the error scores for the RG and BY mechanisms in Figure 3 and Table 2). This lack of consistency between the changes exhibited by both eyes can be seen more easily if we compare the partial total scores for the $\mathrm{RG}$ and $\mathrm{BY}$ mechanisms and the Dain-Birch diagrams from the first and second visits (Figure 3 and Table 1).

The results with the Humphrey perimeter are shown in Figures 9 and 10. There were no changes in the SAP perimetry, but, in the red-on-white perimetry, the size of the central region with sensitivity losses increased. The mfERG result was similar to the one obtained in the first visit (Table 2).

The four ATD perimetry tests for each eye are shown in Figures 11 and 12. For all stimuli, the size of the damaged central region increased, compared with the first visit. With both chromatic stimuli, the damage reached even the periphery of the visual field.

\section{Conclusions}

In this study we examined how chloroquine toxicity affects visual perception, by analyzing the contrast sensitivity losses in different visual mechanisms. We have shown that even after cessation of treatment, the patient's sensitivity steadily worsened, in agreement with previous studies, ${ }^{7,8}$ although not all of the techniques used with our patient were capable of revealing this progression.

We have paid particular attention to the behavior of the chromatic mechanisms. The Farnsworth-Munsell 100-hue test detects global color sensitivity losses affecting both

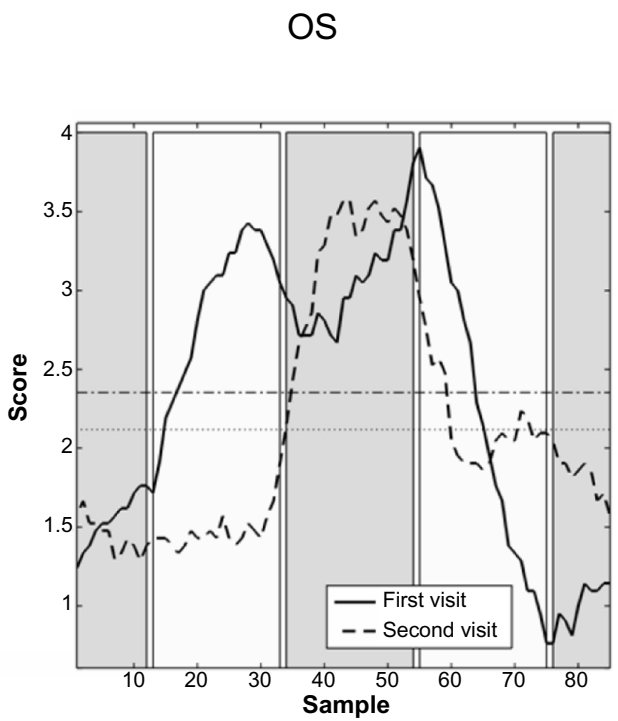

Figure 3 Dain-Birch diagram for Farnsworth-Munsell results at both visits.

Notes: Dark gray regions correspond to the blue-yellow mechanism and light gray to the red-green mechanism. The straight lines represent the mean error, which we have not normalized to I, as proposed by Dain and Birch, in order to be able to compare the two measurements.

Abbreviations: OD, right eye; OS, left eye. 
OD

Total deviation

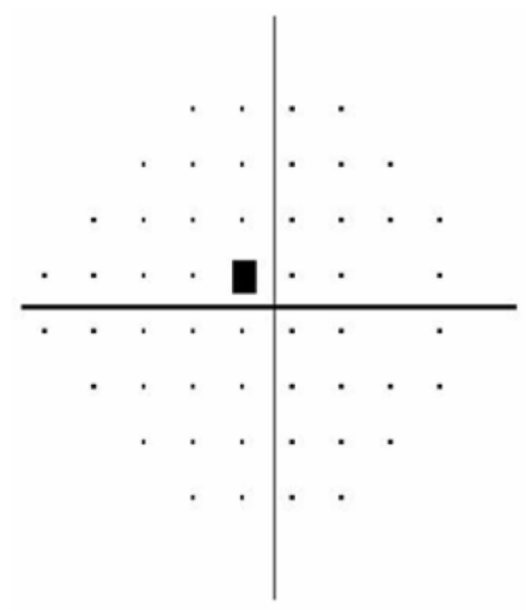

$::<5 \%$

$\%<2 \%$

$<1 \%$

$<0.5 \%$

\section{Pattern deviation}
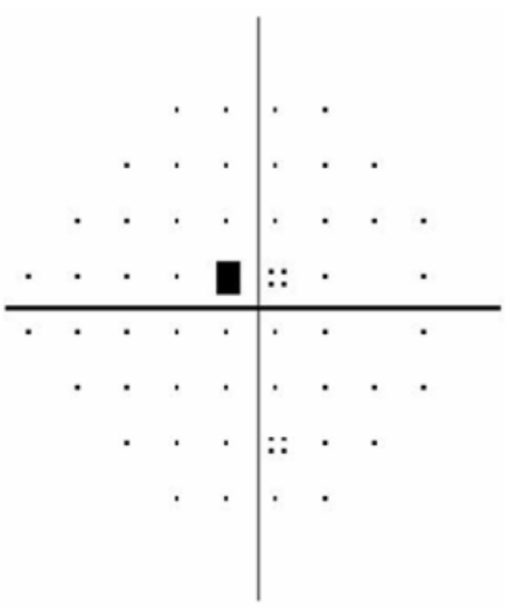

OS

Total deviation

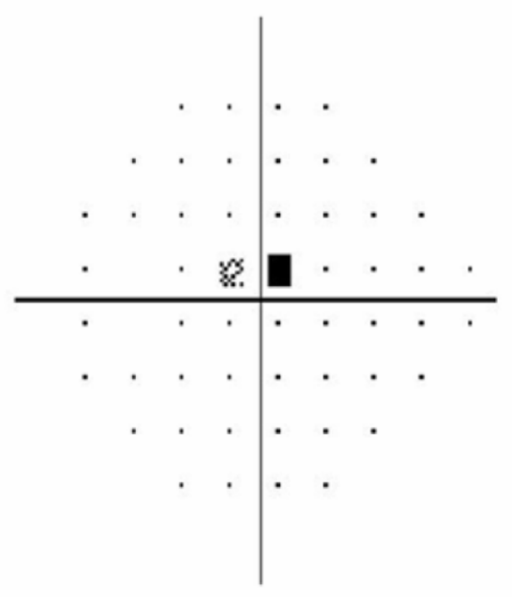

\section{Pattern deviation}

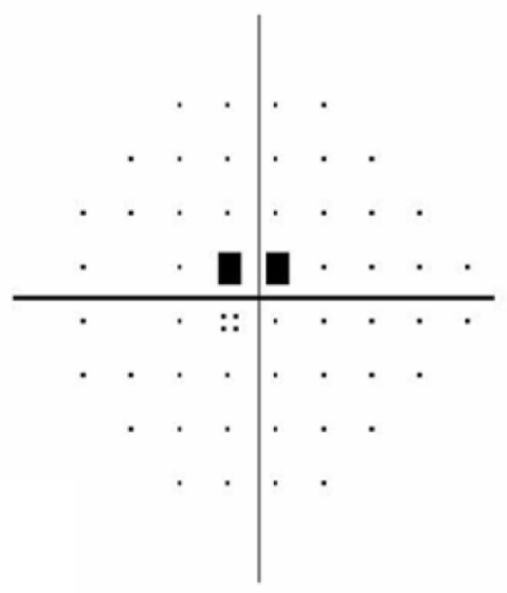

$::<5 \%$

\%. $<2 \%$

亲 $<1 \%$

$<0.5 \%$

Figure 4 Total deviation and pattern deviation maps for SAP Humphrey perimetry on the first visit.

Notes: Top: right eye; Bottom: left eye.

Abbreviations: SAP, standard automated perimetry; OD, right eye; OS, left eye.

the RG and BY mechanisms, but describes a different progression in each eye. In the right eye, the BY mechanism slightly improved and, therefore, the RG component of the defect became more marked, whereas, in the left eye, the defect changed to a selective loss in the BY mechanism. However, the ATD chromatic perimetries show that both the RG and BY mechanisms were affected, and that the extent of visual field where this happened increased with time. This behavior is consistent with the shape of the red-on-white visual fields (Humphrey perimetry), where a depression at the central field was found at both visits.

The perimetry tests for the achromatic mechanisms do not show such extended damage. SAP perimetry does not detect many significant defects in the visual field, and the patient's 
OD

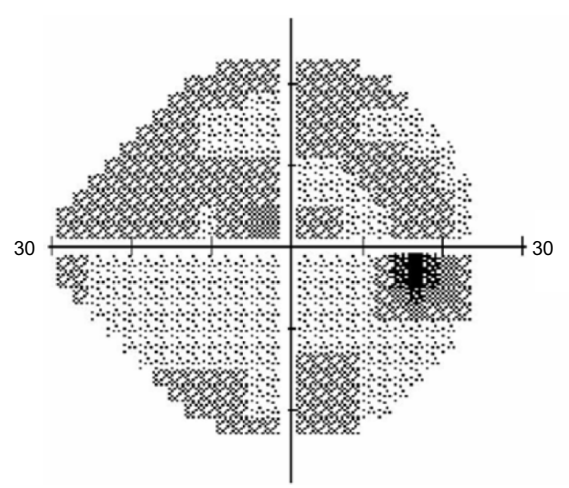

OS

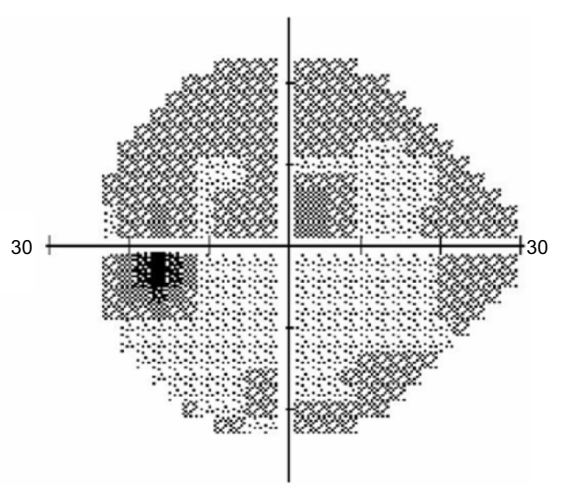

Figure 5 Gray scale sensitivity map for the red-on-white Humphrey perimetry at the first visit. Abbreviations: OD, right eye; OS, left eye.

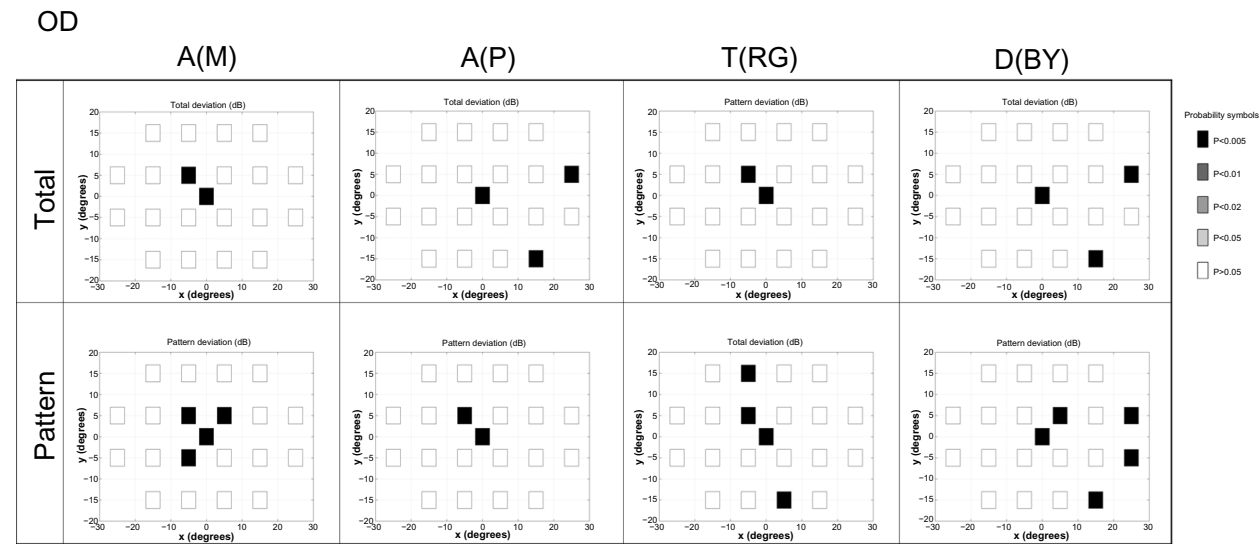

Figure 6 ATD results for right eye.

Notes: $A(M)$ : magnocellular mechanism; $A(P)$ : achromatic parvocellular mechanism: $T(R G)$ : opponent chromatic parvocellular mechanism; $D(B Y)$ : koniocellular mechanism. Top: total deviation map. Bottom: pattern deviation map.

Abbreviations: OD, right eye; A, achromatic; T (or RG), red-green opponent chromatic parvocellular mechanism; D (or BY), blue-yellow koniocellular mechanism green.

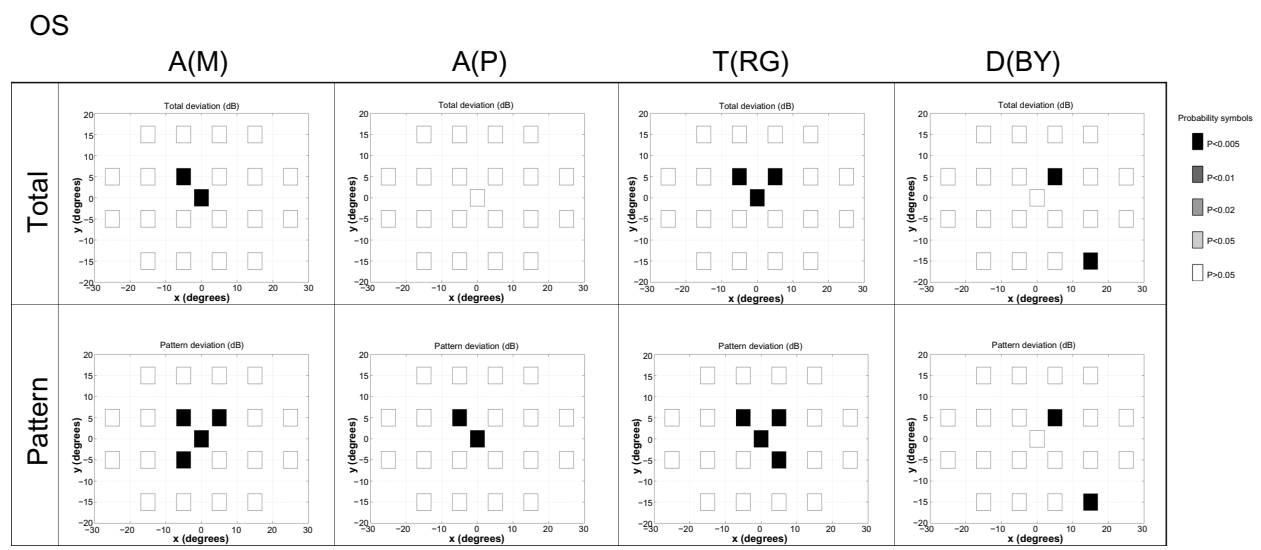

Figure 7 ATD results for left eye.

Notes: $A(M)$ : magnocellular mechanism; $A(P)$ : achromatic parvocellular mechanism: $T(R G)$ : opponent chromatic parvocellular mechanism; $D(B Y)$ : koniocellular mechanism. Top: total deviation map. Bottom: pattern deviation map.

Abbreviations: OS, left eye; A, achromatic; T (or RG), red-green opponent chromatic parvocellular mechanism; D (or BY), blue-yellow koniocellular mechanism green. 
OD

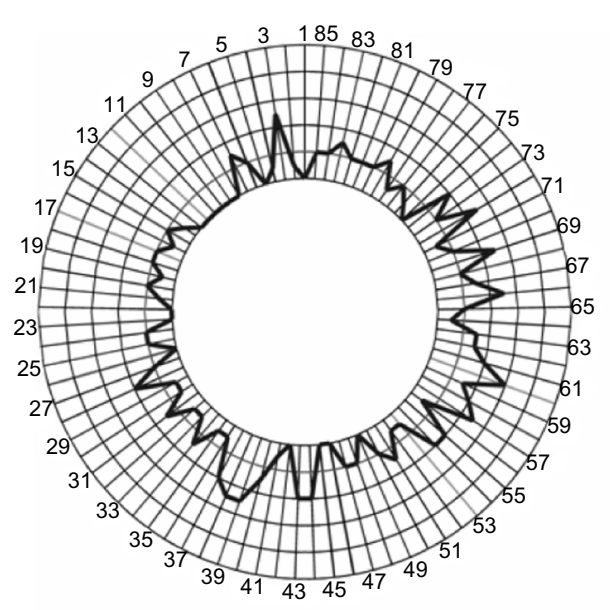

Figure 8 Results for Farnsworth-Munsell 100-hue test on the second visit. Abbreviations: OD, right eye; OS, left eye.

sensitivity at the center of the visual field seemed to improve with time. In the analogue perimetry in the ATD (parvocellular achromatic mechanism), the patient exhibited smaller losses than for the other three stimuli; but, we can observe impairment with time. Two different explanations could be offered for this difference. To begin with, the detectability of the ATD stimulus is smaller than for the Goldman III stimulus used in SAP, and this could highlight subtle defects that go unnoticed by SAP. A further reason may rest on the fact that SAP perimetry is not selective for a particular ganglion cell type.

The debate about the usefulness of visual functionspecific psychophysical tests in detection and follow-up of pathologies is still open. Current opinion is that detectable structural and functional changes can occur concurrently in some patients, whereas either structural or functional change is first apparent in others ${ }^{43}$ Comparison of different psychophysical tests shows that, for a given pathology, not all subjects show damage or appear as normal in the same test types (see, for instance, Sample et $\mathrm{al}^{44}$ ). Even setting aside the objections that can be raised by the procedures used to compare the performance of different techniques, the first difficulty lies in how to ensure that the test really is function-specific (for a review of the hypothesis underlying each test, see Anderson ${ }^{45}$ ). For example, frequency doubling technology perimetry could serve to analyze the magnocellular pathway, high-pass resolution perimetry to analyze the parvocellular pathway, and short wavelength automated perimetry for the koniocellular pathway. This
OS

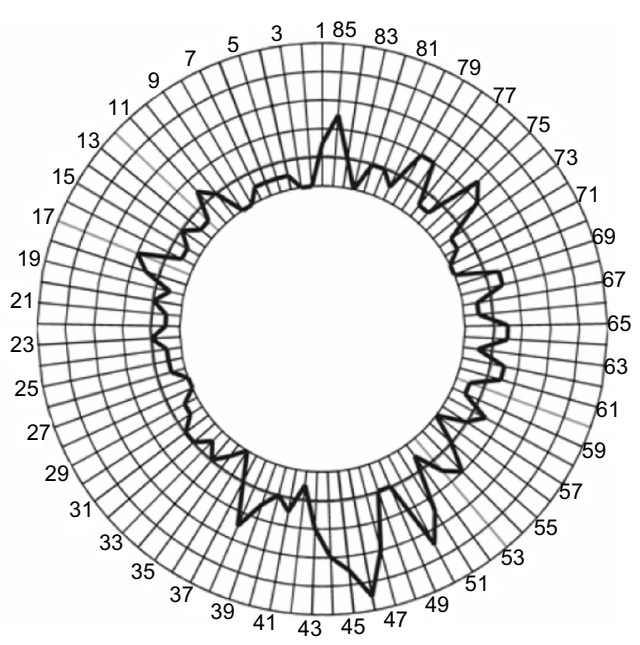

identification of visual tasks with particular cellular pathways is perhaps too simplistic (we refer the interested reader to Kaplan ${ }^{46}$ ). But admitting that these different techniques do favor different mechanisms, even if they are unable to completely isolate them, we find that neither the tasks carried out by the patient nor the metrics used to express the results are comparable, making the analysis of the relative losses incurred by each mechanism difficult. The tests we show in this paper have, at least, the advantage of asking the patient to perform the same kind of task and of using the same metric for all the results..$^{35}$

The case reported in this paper is meant to illustrate the fact that different psychophysical techniques should be used in combination, to gain a clear picture of the visual damage suffered by a particular patient. Even if the pathology is not mechanism-selective, the different visual functions are not equally sensitive to structural damage. ${ }^{44}$ Although structural, objective tests are often preferred by ophthalmologists, it has been argued that psychophysical testing, in spite of different drawbacks, such as variability, is able to detect loss of function, even when the progression of structural damage seems to have been arrested. ${ }^{47}$ The guidelines of the AAO give particular relevance to structural damage and to the functions mediated by the achromatic mechanism; they are concerned with early detection, but not with how to monitor the evolution of visual damage. The role of chromatic vision, either as an early indicator of damage and damage progression or as a key element in the patient's everyday visual tasks, is undervalued. Measuring the sensitivity of 
Table 2 Analysis of the wave PI of the mfERG for both eyes and both visits

\begin{tabular}{llllll}
\hline & \multicolumn{2}{l}{ First visit } & & & \multicolumn{2}{c}{ Second visit } \\
\cline { 2 - 3 } & OD & OS & & OD & OS \\
\hline RI & 24.3 & 35.8 & 44.1 & 20.2 \\
R2 & 14.4 & 11.8 & & 11.5 & 24.7 \\
R3 & 11.0 & 15.6 & 10.7 & 11.4 \\
R4 & 7.5 & 10.2 & 9.2 & 11.9 \\
R5 & 7.6 & 6.3 & & 4.7 & 10.4 \\
R6 & 5.8 & 5.5 & 4.0 & 7.7 \\
\hline
\end{tabular}

Note: We present the density (nV/deg') of each concentric ring (RI-R6).

Abbreviations: OD, right eye; OS, left eye; mfERG, multifocal electroretinogram. different visual pathways could be a good way to help us to understand the visual deficits of these patients, and may help to monitor their evolution.

\section{Acknowledgments}

The ATD multichannel perimeter was built thanks to the support of the Spanish Ministerio de Ciencia y Tecnología (Grants DPI2000-0116-P4-02 and PTR 1995-0909-OP), in collaboration with Industrius De Optica SA (San Cugat del Vallés, Spain).
OD

Total deviation

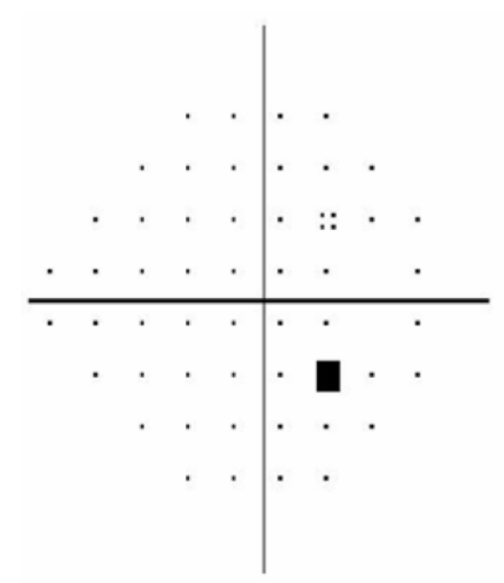

$::<5 \%$

$\%<2 \%$

$<1 \%$

$<0.5 \%$

\section{Pattern deviation}

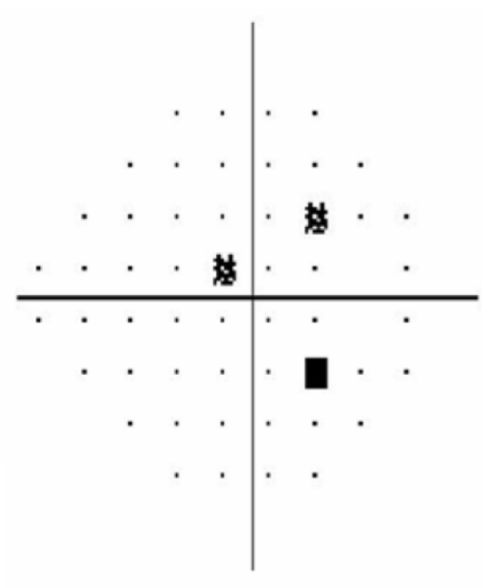

OS

Total deviation

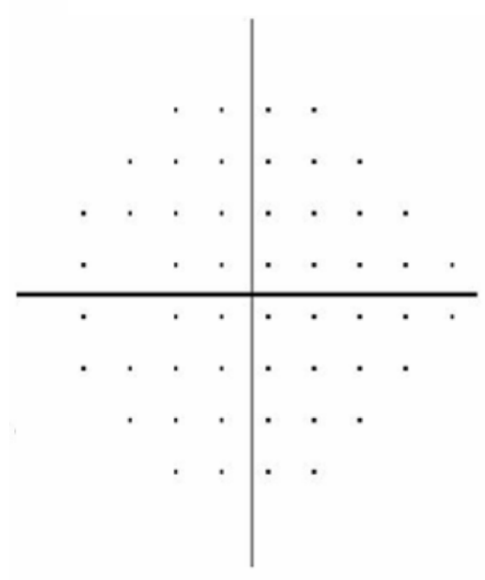

Pattern deviation

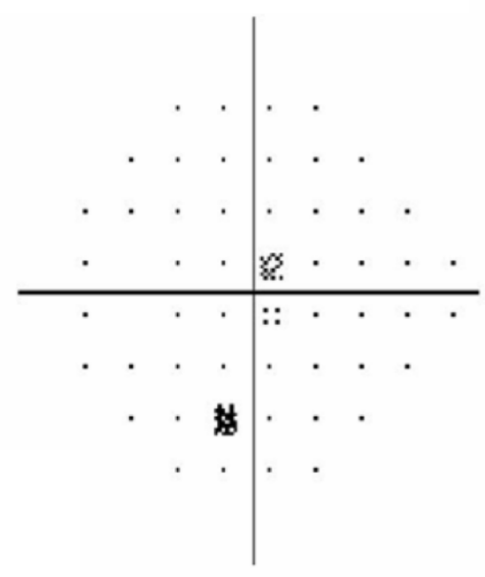

$::<5 \%$

*. $<2 \%$

新 $<1 \%$

$<0.5 \%$

Figure 9 Total deviation and pattern deviation maps for SAP Humphrey perimetry, on the second visit. Abbreviations: SAP, standard automated perimetry; OD, right eye; OS, left eye. 
OD

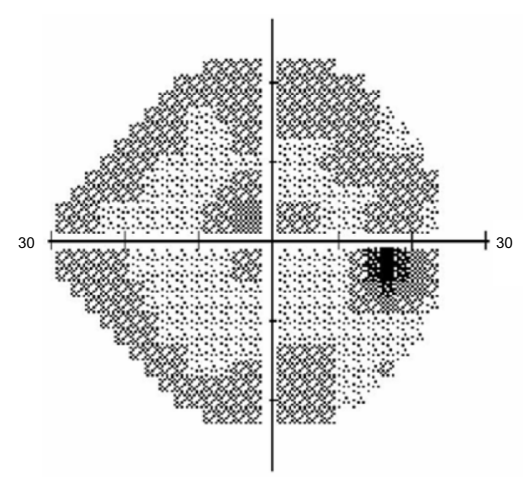

os

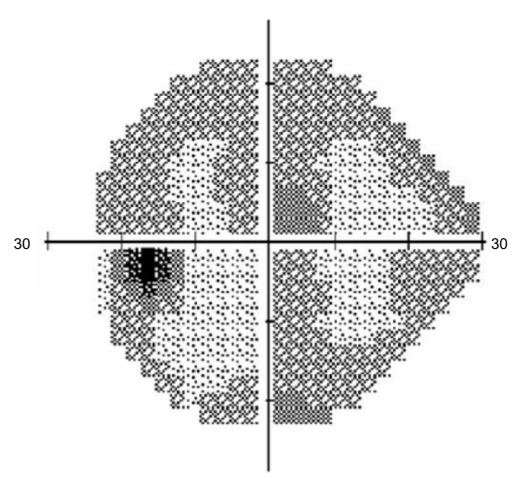

Figure 10 Grayscale sensitivity map for red-on-white Humphrey perimetry, on the second visit. Abbreviations: OD, right eye; OS, left eye.

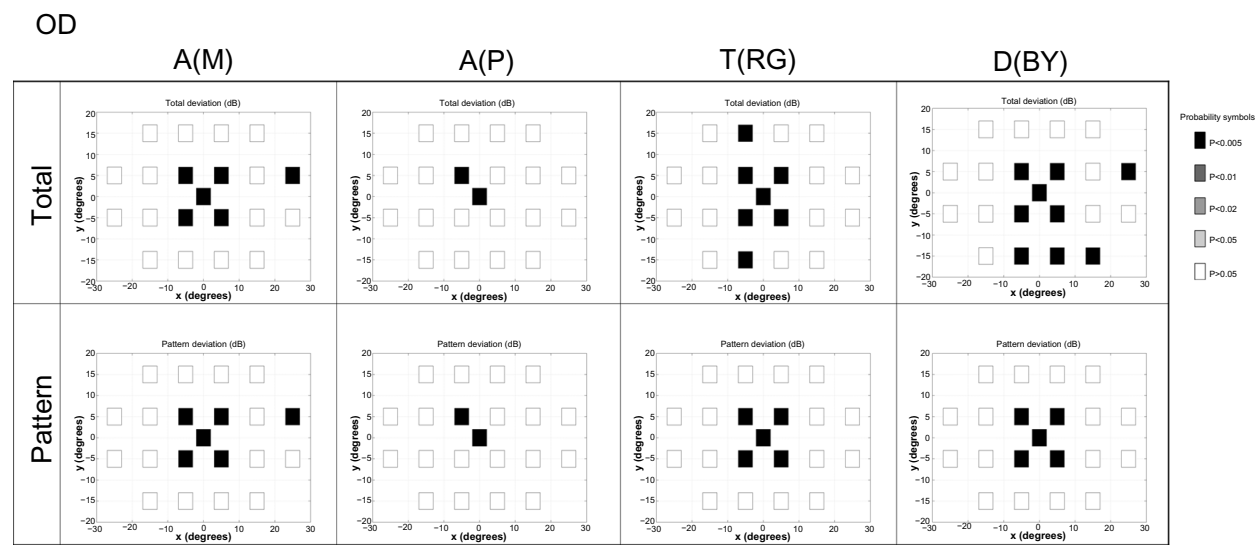

Figure I I ATD results for the right eye.

Notes: $A(M)$ : magnocellular mechanism; $A(P)$ : achromatic parvocellular mechanism; $T(R G)$ : opponent chromatic parvocellular mechanism; $D(B Y)$ : koniocellular mechanism. Top: total deviation map. Bottom: pattern deviation map.

Abbreviations: OD, right eye; A, achromatic; T (or RG), red-green opponent chromatic parvocellular mechanism; D (or BY), blue-yellow koniocellular mechanism green.

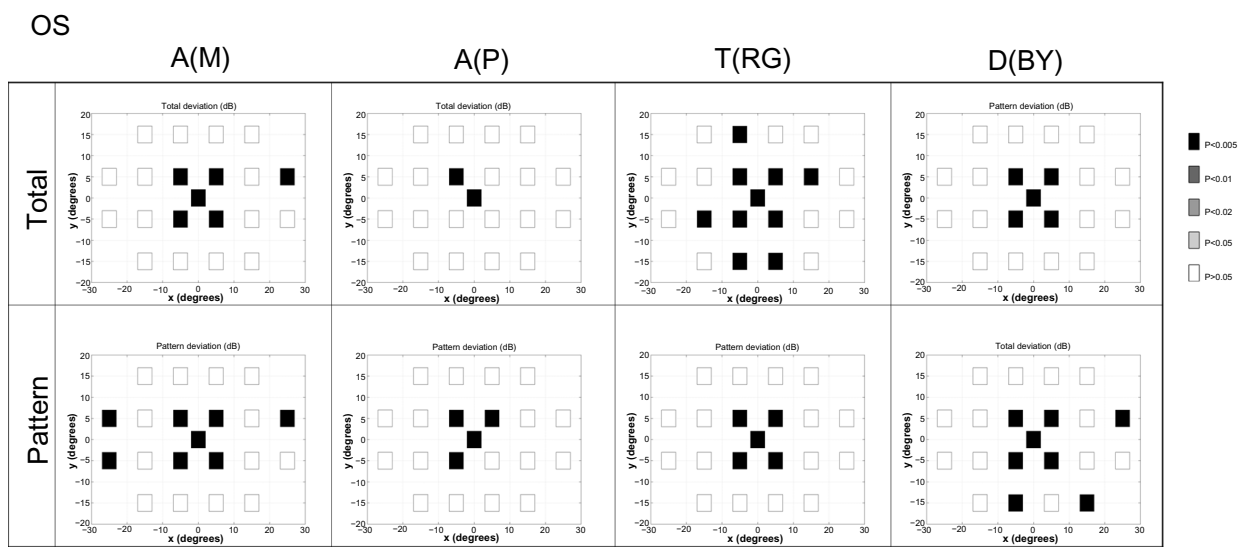

Figure 12 ATD results for the left eye.

Notes: $A(M)$ : magnocellular mechanism; $A(P)$ : achromatic parvocellular mechanism; $T(R G)$ : opponent chromatic parvocellular mechanism; $D(B Y)$ : koniocellular mechanism. Top: total deviation map. Bottom: pattern deviation map.

Abbreviations: OS, left eye; A, achromatic; T (or RG), red-green opponent chromatic parvocellular mechanism; D (or BY), blue-yellow koniocellular mechanism green. 
We wish to thank the Cátedra Alcon-Universidad de Valencia (Valencia, Spain) for their support.

\section{Disclosure}

Preliminary results were presented at the CIOCV'2011 (Internacional de Optometria e Ciências da Visão) conference in Braga, Portugal. Presentation entitled: ATD visual fields versus other clinical proofs in a case of chloroquine retina toxicity. María José Luque has an intellectual proprietary interest in the ATD multichannel perimeter patent (US patent number 7.641.344 B2). María del Carmen García-Domene, María Amparo Díez-Ajenjo, and María Dolores de Fez have no proprietary interest in any material or instrument used in this study.

\section{References}

1. Bryant LR, des Rosier KF, Carpenter MT. Hydroxychloroquine in the treatment of erosive osteoarthritis. J Rheumatol. 1995;22(8):1527-1531.

2. Fox RI, Chan E, Benton L, Fong S, Friedlaender M, Howell FV. Treatment of primary Sjögren's syndrome with hydroxychloroquine. Am J Med. 1988; 85(4A):62-67.

3. Hobbs HE, Sorsby A, Freedman A. Retinopathy following chloroquine therapy. Lancet. 1959; 2(7101):478-480.

4. Cambioggi A. Unusual ocular lesions in a case of systemic lupus erythematosis. Arch Ophthalmol. 1957; 57(3):451-453.

5. Neubauer AS, Samari-Kermani K, Schaller U, Welgi-Lübetaen U, Rudolph G, Berninger I. Detecting chloroquine retinopathy: electro-oculogram versus color vision. $\mathrm{Br} J$ Ophthalmol. 2003; 87(7):902-908.

6. Fielder A, Graham E, Jones S, Silman A, Tullo A. Royal College of Ophthalmologists guidelines: ocular toxicity and hydroxychloroquine. Eye (Lond). 1998;12(Pt 6):907-909.

7. Brinkley JR Jr, Dubois EL, Ryan SJ. Long-term course of chloroquine retinopathy after cessation of medication. Am J Ophthalmol. 1979;88(1): $1-11$.

8. Mavrikakis M, Papazoglou S, Sfikakis PP, Vaiopoulus G, Rougas K. Retinal toxicity in long term hydroxychloroquine treatment. Ann Rheum Dis. 1996;55(3):187-189.

9. Mackenzie AH. Dose refinements in long-term therapy of rheumatoid arthritis with antimalarials. Am J Med. 1983;75(1A):40-45.

10. Marmor MF, Carr RE, Easterbrook M, Farjo AA, Mieler WF. American Academy of Ophthalmology. Recommendations on screening for chloroquine and hydroxychloroquine retinopathy: a report by the American Academy of Ophthalmology. Ophthalmology. 2002;109(7): 1377-1382.

11. Marmor MF, Kellner U, Lai TY, Lyons JS, Mieler WF. American Academy of Ophthalmology. Revised recommendations on screening for chloroquine and hydroxychloroquine retinopathy. Ophthalmology. 2011;118(2):415-422.

12. Browning DJ. Impact of the revised American Academy of Ophthalmology guidelines regarding hydroxychloroquine screening on actual practice. Am J Ophthalmol. 2013;155(3):418-428.

13. Farrell DF. Retinal toxicity to antimalarial drugs: chloroquine and hydroxychloroquine: a neurophysiologic study. Clin Ophthalmol. 2012;6:377-383.

14. Xiaoyun MA, Dongyi HE, Linping HE. Assessing chloroquine toxicity in RA patients using retinal nerve fibre layer thickness, multifocal electroretinography and visual field test. Br J Ophthalmol. 2010;94(12): $1632-1636$.

15. Martínez-Costa L, Victoria Ibañez M, Murcia-Bello C, et al. Use of microperimetry to evaluate hydroxychloroquine and chloroquine retinal toxicity. Can J Ophthalmol. 2013;48(5):400-405.
16. Jivrajka RV, Genead MA, McAnany JJ, Chow CC, Mieler WF. Microperimetric sensitivity in patients on hydroxychloroquine (Plaquenil) therapy. Eye (Lond). 2013;27(9):1044-1052.

17. Okun E, Gouras P, Bernstein H, von Sallmann L. Chloroquine retinopathy: a report of eight cases with ERG and dark-adaptation findings. Arch Ophtalmol. 1963;69:59-71.

18. Nozik RA, Weinstock FJ, Vignos PJ Jr. Ocular complications of chloroquine. A series and case presentation with a simple method for early detection of retinopathy. Am J Ophthalmol. 1964;58:774-778.

19. Bartel PR, Roux P, Robinson E, et al. Visual function and long-term chloroquine treatment. S Afr Med J. 1994;84(1):32-34.

20. Carr RE, Gouras P, Gunkel RD. Chloroquine retinopathy. Early detection by retinal threshold test. Arch Ophthalmol. 1966;75(9):171-178.

21. Easterbrook M. Ocular effects and safety of antimalarial agents. Am J Med. 1988;85(4A):23-29.

22. Henkind P, Carr RE, Siegel IM. Early chloroquine retinopathy: clinical and functional findings. Arch Ophtalmol. 1964;71:157-165.

23. Nylander U. Ocular damage in chloroquine therapy. Acta Ophthalmol. (Copenh). 1966;44(3):335-348.

24. Percival SP, Meanock I. Chloroquine: ophthalmological safety, and clinical assessment in rheumatoid arthritis. Br Med J. 1968;3(6618): 579-584.

25. Grutzner P. Acquired color vision defects secondary to retinal drug toxicity. Ophthalmologica. 1969;158 Suppl:592-604.

26. Jaeger W. [Acquired color-vision-deficiencies caused by side-effects of pharmacotherapy]. Klin Monatsbl Augenheilkd. 1977;170(3):453-460. German.

27. Vu BL, Easterbrook M, Hovis JK. Detection of color vision defects in chloroquine retinopathy. Ophthalmology. 1999;106(9):1799-1803; discussion 1804.

28. Merigan WH, Byrne CE, Maunsell JH. Does primate motion perception depend on the magnocellular pathway? J Neurosci. 1991;11(11): $3422-3429$.

29. Merigan WH, Eskin TA. Spatiotemporal vision of macaques with severe loss of P beta retinal ganglion cells. Vision Res. 1986;26(11): $1751-1761$.

30. Merigan WH, Maunsell JH. Macaque vision after magnocellular lateral geniculate lesions. Vis Neurosci. 1990;5(4):347-352.

31. Merigan WH. P and M pathway specialization in the macaque. In: Valberg A, Lee BB, editors. From Pigments to perception. New York: Plenum; 1991.

32. Schiller PH, Logothetis NK, Charles ER. Functions of the coloropponent and broad-band channels of the visual system. Nature. 1990 343(6253):68-70.

33. Hendry SH, Yoshioka T. A neurochemically distinct third channel in the macaque dorsal lateral geniculate nucleus. Science. 1994;264(5158): 575-577.

34. Capilla P, Luque MJ, de Fez MD, García-Domene MC, Díez-Ajenjo MA. Simulating images seen by patients with inhomogeneous sensitivity losses. Optom Vis Sci. 2012;89(10):1543-1556.

35. Anton A, Capilla A, Morilla-Grasa A, Luque MJ, Artigas JM, Felipe A. Multichannel functional testing in normal subjects, glaucoma suspects and glaucoma patients. Invest Ophtalmol Vis Sci. 2012;53(13): 8386-8395.

36. Díez-Ajenjo MA, Capilla P, Luque MJ. Red-green vs blue-yellow spatiotemporal contrast sensitivity across the visual field. Journal of Modern Optics. 2011;58:1736-1748.

37. Birch J, Dain SJ. An averaging method for the interpretation of the Farnsworth-Munsell 100-hue test - II. Color vision defects acquired in diabetic retinopathy. Ophthalmic Physiol Opt. 1987;7(3): 281-291.

38. Dain SJ, Birch J. An averaging method for the interpretation of the Farnsworth-Munsell 100-hue test - I. Congenital color vision defects. Ophthalmic Physiol Opt. 1987;7(3):267-280.

39. Smith VC, Pokorny J, Pass AS. Color-axis determination on the Farnsworth-Munsell 100-hue test. Am J Ophthalmol. 1985; 100(1):176-82. 
40. Kinnear PR, Sahraie A. New Farnsworth-Munsel 100 hue test norms of normal observers for each year of age 5-22 and for age decades 30-70. Br J Ophthalmol. 2002;86(12):1408-1411.

41. Brainard DH. Cone contrast and opponent modulation color spaces. In: Kaiser PK, Boynton RM, editors. Human Color Vision. Washington, DC: Optical Society of America; 1996;563-579.

42. Derrington AM, Krauskopf J, Lennie P. Chromatic mechanisms in lateral geniculate nucleus of macaque. J. Phisyol. 1984;357:241-265.

43. Garway-Heath DF, McNaught A, Jansonius N, et al. Visual function progression. In: Weinreb RN, Garway-Heath DF, Leung C, Crowston JG, Medeiros FA, editors. Progression of glaucoma. Amsterdam, Netherlands: Kugler Publications; 2011.
44. Sample PA, Medeiros FA, Racette L, et al. Identifying glaucomatous vision loss with visual-function specific perimetry in the diagnostic innovations in glaucoma study. Invest Ophthalmol Vis Sci. 2006;47(8): 3381-3389.

45. Anderson RS. The psychophysics of glaucoma: improving the structure/ function relationship. Prog Retin Eye Res. 2006;25(1):79-97.

46. Kaplan E. The M, K, and P streams in the primate visual system: what do they do for vision?. In: Masland R, Albright T, editors. The senses: a comprehensive reference. London: Elsevier; 2006;369-382.

47. Sample PA. What does functional testing tell us about optic nerve damage? Surv Ophthalmol. 2001;45 Suppl 3:S3192-S324; discussion S332-S334.
Clinical Optometry

\section{Publish your work in this journal}

Clinical Optometry is an international, peer-reviewed, open access journal publishing original research, basic science, clinical and epidemiological studies, reviews and evaluations on clinical optometry. All aspects of patient care are addressed within the journal as well as the practice of optometry including economic and business analyses. Basic and clinical

Submit your manuscript here: http://www.dovepress.com/clinical-optometry-journal

\section{Dovepress}

research papers are published that cover all aspects of optics, refraction and its application to the theory and practice of optometry. The manuscript management system is completely online and includes a very quick and fair peer-review system, which is all easy to use. Visit http://www.dovepress. com/testimonials.php to read real quotes from published authors. 